\title{
A Trajectory Based Methods for Parallel Scheduling of Machines and AGV'S in an FMS Environment
}

\author{
P.Sreenivas ${ }^{1}$, Shaik khajapeer saheb ${ }^{2}$, M.Yohan ${ }^{3}$ \\ ${ }^{1}$ Research Scholar, Dept. of Mechanical Engineering, JNTUA College of Engineering- \\ Ananthapuramu, Andhra Pradesh, India. \\ ${ }^{2}$ Professor \& Principal, KLM College of Engineering for Women-Kadapa, Andhra Pradesh, India. \\ ${ }^{3}$ Professor, Dept. Of Mechanical Engineering, JNTUA College of Engineering-Ananthapuramu, \\ Andhra Pradesh, India.
}

\begin{abstract}
Now a days latest manufacturing sectors facing difficulty with two different categories of processing of parts like semi-finished jobs and finished jobs. In literature it is assumed that, for machine scheduling models, either there is infinite number of handling or carrier systems for the job to reach desired location or jobs are distributed on the spot from one workstation to other without travel time involved. Further it is observed that simultaneous scheduling of machines and AGVs in the FMS environments involves certain exceptional difficulties as they are treated as NP hard problems. Those difficulties are assigning AGV in sequence of task along with operation sequence of machines and operation order of machines based on precedence constraints. Trajectory based methods are powerful metaheuristic for solving combinatorial optimization problems like NP hard problems such as scheduling. In this paper, author addressed about parallel scheduling of machines and AGV's for determining minimum makespan, mean makespan and tardiness using simulated annealing algorithm and tested over 20 standard benchmark problems collected from literature. Finally, the proposed method is compared with literature results and conclusions are depicted.
\end{abstract}

Keywords: Flexible manufacturing system, NP hard problems, Parallel scheduling, Makespan, Tardiness Simulated annealing algorithm

\section{Introduction}

Flexible manufacturing systems (fms) are intended to merge the effectiveness of a massproduction and the flexibility of a job shop for the batch production of a part variety and mix of products. fmss enlarge the flexibility and productivity of distinct part manufacturing. it is observed that fms is not only fetching complexity to control, but also caters a number of decision problems. the nature of a fms is entirely different from a conventional job shop. due to new circumstances of fms over job shop, it caters new capabilities which give new constraints to the scheduling problem. the flexibility in manufacturing can be observed by free movement of parts with in shop floor without any delay from one station to other based on hardware and layout design in fms. further, setup time of machine is assumed to be zero. so, machines can be switch over to part to part with zero down time.

During former epoch, there was a considerable research has been made to scheduling of machines and agv's separately in fms environment, despite the two problems are closely interconnected. literature survey shows that only few researchers addressed the scheduling of agv's and machines as one stream. this area of research have many difficulties such as precedence constraints of travel times dead heading trips between successive loaded trips of agvs. hence, this paper aims at np hard problems by introducing heuristic algorithm which involves both agv and 
machine scheduling constraints with an objective of makespan minimization and determination of mean makespan and tardiness.

\section{Literature Survey}

Now a days, owing to huge capital investment and also having high potential FMS act as tactical aggressive tool which makes an attractive subject for researchers (1). Based on this, researchers have one more attractive area in FMS is parallel scheduling of machines and AGV's which was addressed by few researchers in (2) various ways for formulation of online priority dispatche rules. It is observed that FMS scheduling has been widely used over last three decades which sequentially gains the interest of both industrial and academic sectors. (3) focused on Biobjective scheduling problem in flexible manufacturing cell in order to determine maximum completion time (makespan) and maximum tardiness.They proposed a new hybrid genetic algorithm (HGA) combined with four priority dispatching rules. (4) addressed the logistic scheduling refers to problems inwhich decisions on job scheduling and transportation are integrated into a single framework.A logistics scheduling model for two processing centers that are located in different cities is presented. Author have considered two different transshipment modes:

(i) item transshipment;

(ii) batch transshipment

Under item transshipment, once a job in Bik is completed in center $M k$, it is immediately transported to center $M i$. In batch transshipment, all jobs in Bik have to be transported together as a batch from $M k$ to $M i$ when the last jobin ${ }^{-} B i k$ is completed on $M k$.(5) proposed a multiagent based scheduling approach for AGVs and machines within a manufacturing system where the AGV breakdowns are considered. Authors focused on AGV breakdown situation modeling under a multiagent based system approach. The proposed method is designed by using the Prometheus methodology that defines a detailed process for specifying, designing, implementing, and testing/debugging agent oriented software systems. (6) addresses the simultaneous job input sequencing and vehicle dispatching problems in flexible manufacturing systems (FMS) using a single device Automated Guided Vehicle System (AGVS).They proposed a new lower bound and tested the benchmarks stating that this lower bound Outper forms the lower bound previously published.

\section{Mathematical Formulation}

Given an FMS environment with workstations, AGVs and set of jobs, determine the starting and completion times of operations for each job and the trips between workstations together with the vehicle assignment. The objective is to minimize the makespan (Cmax) which implies that the cost of a schedule depends on the duration for which the whole system is allocated to process a set of jobs.

3.1 Objective Function

$1 \quad$ Minimize $($ Makespan $)=\operatorname{Max}(\mathrm{C} 1, \mathrm{C} 2, \mathrm{C} 3 \ldots \mathrm{Cn})$

$$
\mathrm{n}
$$

$$
\text { Job Completion Time }=\mathbf{C}_{\mathbf{i}}=\square \quad \mathrm{O}_{\mathrm{ij}}
$$$$
\mathrm{i}=1
$$

Operation completion time $=\mathrm{O}_{\mathrm{ij}}=\mathrm{T}_{\mathrm{ij}}+\mathrm{P}_{\mathrm{ij}}$

2. Tardiness $=\mathrm{T}_{\mathrm{i}}=\mathrm{C}_{\mathrm{i}}-\mathrm{D}_{\mathrm{i}}$

$$
\mathrm{n}
$$

3. Mean Tardiness $=(1 / \mathrm{n}) \sum \mathrm{T}_{\mathrm{i}}$

$$
\mathrm{i}=1
$$




\section{Configuration And Operating Environment of FMS}

a

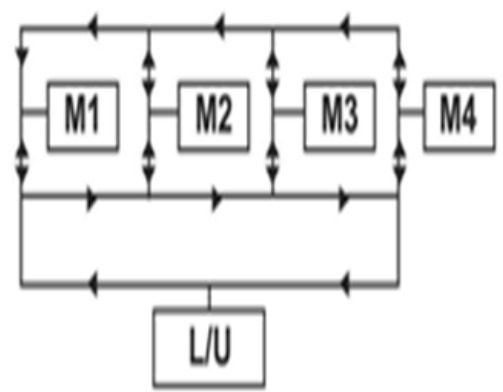

Figure1: a) FMS layout-1 b

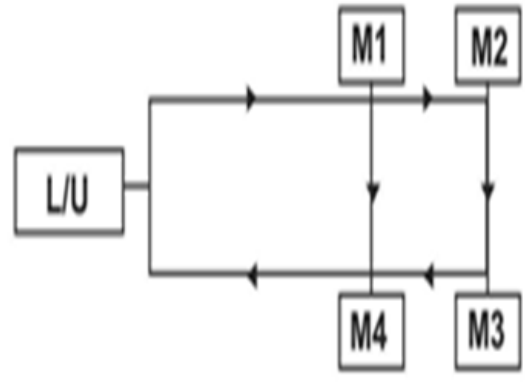

b) FMS layout2

\section{FMS Description}

Today Flexible Manufacturing System (FMS) seem to be a very promising technology as they provide a variety of flexibility that is essential for design of planning for simultaneous scheduling of machines and automated guided vehicles (AGVs) to stay competitive in the highly dynamic and changing design environment. In the literature reported that, the subject of design of planning for simultaneous scheduling of machines and automated guided vehicles (AGVs) using non optimization technique system has generally been set out either as a comparison of various vehicle dispatching rules in relation to a prespecified schedule and on a particular layout $[7,8]$ or in relation with the design jobset $[9,10]$.

\subsection{Proposed Methodology:}

In this study, a flexible manufacturing system (FMS) in which material transfer between machines is performed by a number of identical automated guided vehicles (AGVs) is considered, and the problem of design of planning for simultaneous scheduling of machines and AGVs using nontraditional optimization technique is addressed. Authors have considered 4 different layouts and 5 job sets consisting of 1-10 different job sets and operations on machines to be performed. The problem objective is makespan minimization, mean makespan, mean tardiness and CPU time. The formulation consists of constraint sets of a machine scheduling sub problem and a vehicle scheduling sub problem which interact through a set of Tabu search and simulated algorithm constraints for the material handling trip starting times. An iterative procedure is developed where, at each iteration, a new machine schedule is generated by a Tabu search and simulated algorithm procedure.

\subsection{Tabu Search:}

Tabu search is a higher level search technique which directs the natural search procedure to survey the solution space beyond local optimality. Here, the key components of Tabu search is its concern with adapting memory, that enables the procedure search behavior with more flexibility.TS is depends on the vicinity of problem, incorporate adaptive memory and receptive exploration. Being more concerned in adapting memory features, TS gives provision for applying the procedures that are able to search the solution space economically and effectively. It is known that local selections are directed by information gathered during the search, TS shows difference with memory less designs which greatly depends up on semi random processes that employ a form of sampling. 


\subsection{Simulated Annealing:}

Simulated Annealing is a trajectory based probabilistic method introduced by Kirkpatric, Gelett and Vecchi (1983) and cerny (1985) for identifying the global[20] minimum of a objective function which have several local minima. It works on the principle of emulating the physical annealing process in which solid is slowly cooled so that finally its structure is "frozen" this will happens at a minimum energy system. Annealing is a physical process for obtaining a low energy state of a solid in two steps:

1. Attaining recrystalization point while heating metal.

2 Reducing temperature of the metal slowly by cooling, allowing it to attain thermal balance at each temperature.

\section{Results And Comparison}

Trajectory based algorithm procedure has been used for generating necessary code in C++ language and simulation is done using IDE tool for various test problems. As per the AGV assignment heuristics for different trajectory based algorithms, the simulation code is developed. Here population size is considered as 1 due to trajectory based nature of algorithms which means having only one population in each generation.

The authors have considered SET of problem instances. The SET of problems is designed as Problem instances $1-2$, here 1 indicates layout 1 problems and 2 indicates layout 2 problems. Under the problem instances $1-2$ the designations are indicated as INST XY. Where X stands for layout number and y stands for job set number. Table 1 shows that the results of make span for $t / p$ ratio > 0.25 and these results are executed for 100 generation on IDE Simulation software in each trail for which 10 trail runs are conducted for proposed trajectory based algorithms. The bench mark problems are solve through the propose algorithms and the results are assimilated and observed that for all problem instances the makespan value is higher at initial trail and reach to minimum value at final trail and gets fluctuate in intermediate trails.

Table 1 :- Comparison of MEAN MAKESPAN results of the trajectory based method for $t / p>0.25$

(Problem instances 1-2) with 100 generations for 10 trials

\begin{tabular}{|c|c|c|c|c|c|c|c|c|c|c|c|c|c|c|c|c|c|c|c|c|}
\hline \multirow{2}{*}{$\begin{array}{l}\text { PROB } \\
\text { INST }\end{array}$} & \multicolumn{10}{|l|}{ SA } & \multicolumn{10}{|l|}{ TS } \\
\hline & T1 & $\mathrm{T} 2$ & T3 & T4 & T5 & T6 & T7 & T8 & T9 & T10 & T1 & $\mathrm{T} 2$ & T3 & T4 & T5 & T6 & T7 & T8 & T9 & T10 \\
\hline INST11 & 68 & 66 & 59 & 59 & 57 & 64 & 68 & 68 & 62 & 52 & 67 & 77 & 68 & 62 & 69 & 62 & 64 & 62 & 71 & 62 \\
\hline INST12 & 65 & 62 & 65 & 63 & 58 & 65 & 66 & 68 & 65 & 62 & 65 & 68 & 66 & 61 & 67 & 67 & 67 & 64 & 61 & 58 \\
\hline INST13 & 73 & 68 & 65 & 75 & 75 & 72 & 76 & 73 & 71 & 61 & 71 & 77 & 70 & 69 & 67 & 76 & 68 & 71 & 69 & 63 \\
\hline INST14 & 70 & 64 & 68 & 63 & 66 & 69 & 70 & 63 & 65 & 63 & 66 & 72 & 67 & 70 & 70 & 74 & 68 & 69 & 64 & 64 \\
\hline INST15 & 60 & 51 & 47 & 47 & 48 & 49 & 60 & 53 & 55 & 44 & 48 & 58 & 55 & 52 & 60 & 49 & 52 & 52 & 48 & 46 \\
\hline INST16 & 70 & 74 & 70 & 71 & 71 & 71 & 75 & 67 & 71 & 61 & 69 & 74 & 73 & 71 & 67 & 73 & 71 & 73 & 67 & 66 \\
\hline INST17 & 54 & 54 & 59 & 54 & 56 & 58 & 59 & 55 & 60 & 49 & 56 & 58 & 55 & 54 & 54 & 54 & 53 & 59 & 56 & 51 \\
\hline INST18 & 79 & 81 & 86 & 74 & 82 & 86 & 86 & 84 & 86 & 80 & 86 & 90 & 81 & 82 & 81 & 81 & 79 & 86 & 82 & 77 \\
\hline INST19 & 68 & 65 & 65 & 65 & 65 & 70 & 72 & 64 & 64 & 63 & 64 & 66 & 65 & 65 & 64 & 65 & 63 & 65 & 66 & 63 \\
\hline INST110 & 75 & 80 & 76 & 79 & 76 & 76 & 80 & 76 & 74 & 72 & 79 & 79 & 78 & 71 & 74 & 77 & 77 & 76 & 72 & 67 \\
\hline INST21 & 50 & 60 & 60 & 60 & 50 & 56 & 60 & 58 & 52 & 48 & 56 & 60 & 60 & 60 & 55 & 50 & 42 & 54 & 57 & 44 \\
\hline INST22 & 62 & 64 & 66 & 62 & 64 & 64 & 67 & 62 & 61 & 56 & 63 & 67 & 63 & 60 & 61 & 57 & 61 & 61 & 63 & 52 \\
\hline INST23 & 65 & 65 & 67 & 66 & 66 & 66 & 70 & 66 & 67 & 57 & 58 & 70 & 65 & 69 & 69 & 65 & 63 & 61 & 59 & 57 \\
\hline INST24 & 60 & 56 & 58 & 58 & 52 & 60 & 60 & 55 & 56 & 55 & 60 & 60 & 57 & 56 & 60 & 56 & 59 & 50 & 55 & 55 \\
\hline INST25 & 42 & 42 & 42 & 50 & 46 & 42 & 46 & 45 & 45 & 42 & 45 & 48 & 45 & 42 & 42 & 48 & 41 & 46 & 48 & 37 \\
\hline INST26 & 75 & 72 & 73 & 76 & 75 & 75 & 76 & 73 & 73 & 68 & 73 & 80 & 72 & 74 & 68 & 72 & 73 & 72 & 76 & 67 \\
\hline INST27 & 59 & 52 & 56 & 56 & 57 & 52 & 61 & 60 & 58 & 46 & 56 & 59 & 56 & 59 & 57 & 57 & 54 & 57 & 57 & 52 \\
\hline INST28 & 79 & 78 & 76 & 74 & 78 & 77 & 82 & 77 & 76 & 76 & 77 & 81 & 81 & 79 & 79 & 81 & 77 & 81 & 79 & 74 \\
\hline INST29 & 63 & 63 & 53 & 65 & 65 & 63 & 64 & 62 & 63 & 62 & 63 & 65 & 63 & 63 & 63 & 63 & 63 & 62 & 63 & 58 \\
\hline INST2 10 & 72 & 68 & 71 & 73 & 74 & 73 & 75 & 71 & 73 & 66 & 73 & 78 & 75 & 73 & 73 & 71 & 73 & 73 & 69 & 65 \\
\hline
\end{tabular}




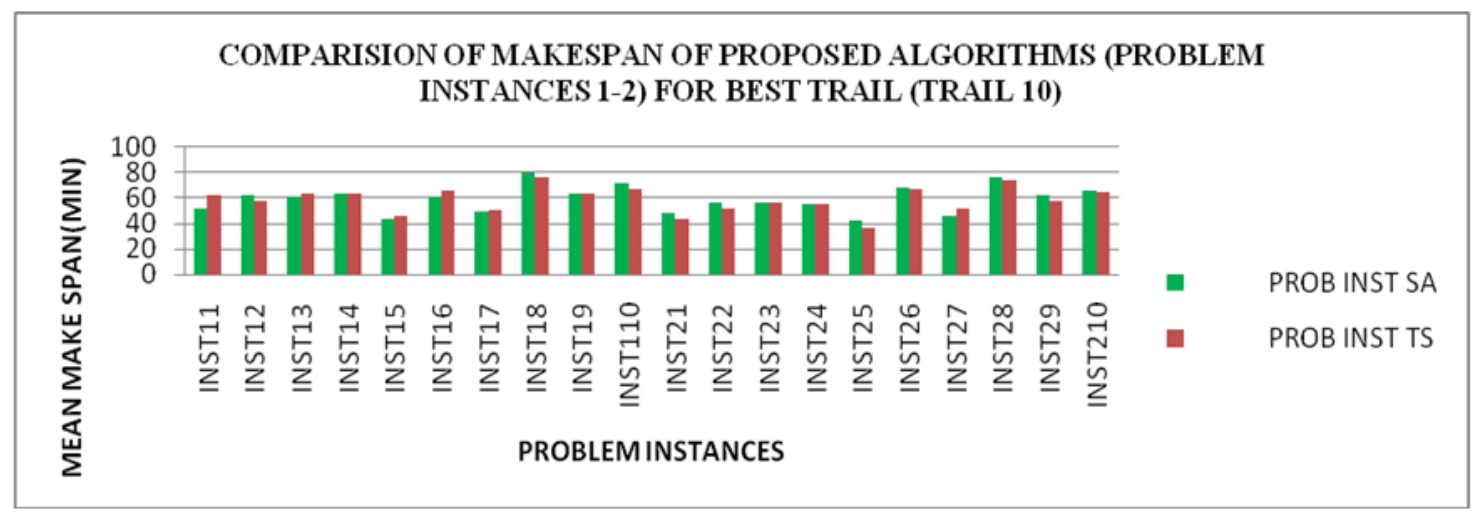

Fig 2: Comparision of Makespan of Proposed Algorithms (Problem Instances 1-2) For Best Trail (Trail 10)

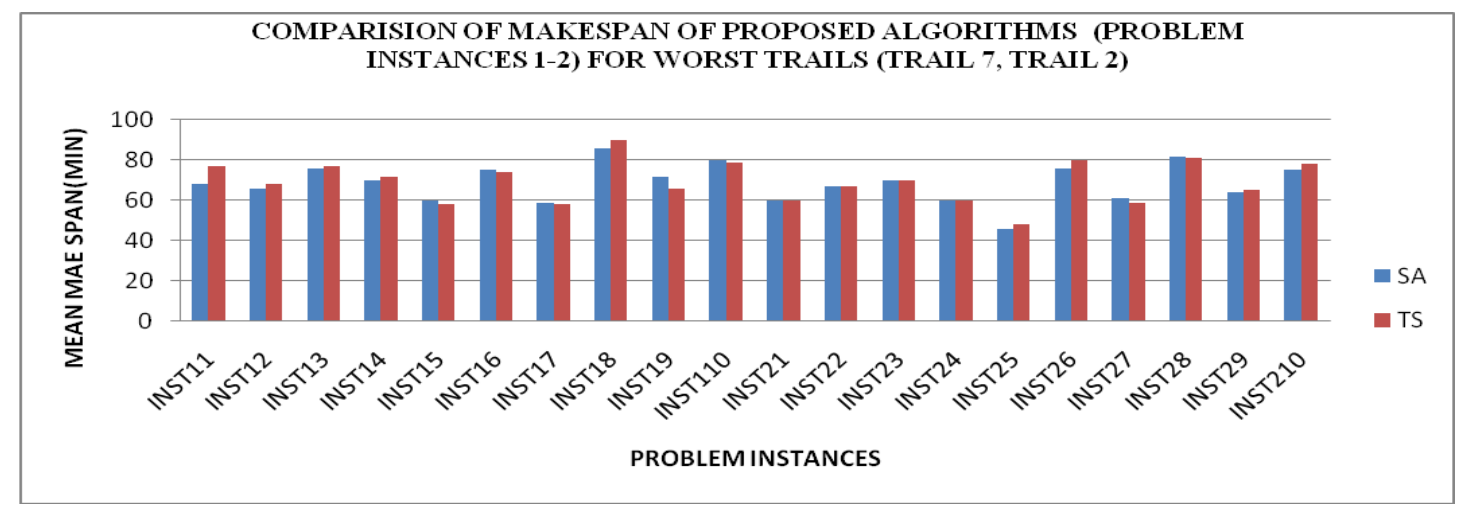

Fig 3: Comparision Of Makespan Of Proposed Algorithms (Problem Instances 1-2) For Worst Trails (Trail 7, Trail 2)

The graphs shown in Figs. 2 and 3 are styled for problem instances1-2. It is observed that there are moderate variations in results of Mean makespan against problem instances shown in the plot for SA and TS. It is found that Mean makespan is low at problem instance INST25 and generates high Mean makespan value for INST18 whereas at remaining problems. Mean makespan varies moderately because it depends on size of the problem. Furthermore, SA provides marginally better values than TS curve, since Simulated Annealing is probabilistic metaheuristic which can search for more feasible solutions by cooling probability where as Tabu search does not have such parameters.

\section{Conclusions}

In this paper, it is observed that the proposed trajectory based algorithm needs two stages of calculation. During the inception of algorithms as first stage, it is compulsory to recognize the nature of algorithms and their probabilities in finding the optimum solution. While in the second stage, order of machines are altered and accordingly machine representations act as data flow into the trajectory based algorithm by accepting and rejecting based on precedence constraints in yielding optimum solutions. The performance of the proposed algorithm is tested over a number of problems selected from the literature and is compared to many other algorithms existing in the literature. Hence from the results and discussion it is understand that for best trail, out of proposed trajectory based method, Tabu search performance is marginally better than simulated annealing for layout 2 and 4 where as for layout 1 both methods are performing same manner in yielding mean makespan. On other side for worst trail, Tabu search provides marginally better mean makespan results than simulated annealing for layout 2 where as for layout 1 both methods are equally works in getting the results. 


\section{References}

[1]. Jerald, J., \& Asokan, P. (2006). Simultaneous scheduling of parts and automated guided vehicles in an FMS environment using adaptive genetic algorithm. Int J Adv Manuf Technol (2006) 29: 584-589. DOI 10.1007/s00170005-2529-9

[2]. Satish Kumar, M. V., \& Rao. C.S.P.(2011). Simultaneous scheduling of machines and vehicles in an FMS environment with alternative routing. Int J Adv Manuf Technol (2011) 53:339-351 DOI 10.1007/s00170-010-2820-2

[3]. R. Tavakkoli-Moghaddam \& M. Heydar.(2013) A hybrid GA for simultaneously scheduling an FMC under multiple objectives. 5th International Conference on Applied Operational Research, Proceedings, Vol. 5: 133-142

[4]. XIANGTONG QI..(2006). A logistics scheduling model: scheduling and transshipment for two processing centers. IIE Transactions (2006) 38, 609-618, DOI: 10.1080/074081791009022

[5]. Vahit Kaplanoglu\& Cenk Sahi.(2014) . A Multi-Agent Based Approach to Dynamic Scheduling of Machines and Automated Guided Vehicles (AGV) in Manufacturing Systems by Considering AGV Breakdowns . Proceedings of The 2014 IAJC-ISAM International Conference, ISBN 978-1-60643-379-9

[6]. Lacomme. P \& Moukrim A .(2014). A new lower bound for scheduling of fms based on agv Material handling. 15th Triennial World Congress, Barcelona, Spain

[7]. K. V. Subbaiah, M. Nageswara Rao and K. Narayana Rao "Scheduling of AGVs and machines in FMS with make span criteria using sheep flock heredity algorithm”. International Journal of Physical Sciences Vol. 4(2), pp. 139-148, March, 2009.

[8]. A. Chaudhry, S. Mahmood, M. Shami "Simultaneous scheduling of machines and automated guided vehicles in flexible manufacturing systems using genetic algorithms". National University of Sciences \& Technology (NUST), Islamabad, PAKISTAN.

[9]. Dr. T. Ghose. "Optimization Techniques and An introduction to genetic algorithms and simulated annealing". Dept. of EEE, BIT, Mesra.

[10]. D. Banerjee and R. Bhattacharya "Robust Design of an FMS and Performance Evaluation of AGVs". Production Engineering Department, Jadavpur University, Kolkata-700032, India 